\title{
INTOXICAÇÃO MEDICAMENTOSA INFANTIL NO PARANÁ
}

\author{
DRUG INTOXICATION IN CHILDREN IN PARANÁ
}

\section{Thiago José da SILVA'; Vinícius Bednarczuk de OLIVEIRA²}

\begin{abstract}
1 - Aluno do curso de Farmácia da Uniandrade, Curitiba - PR, Brasil.
2 - Professor do curso de Farmácia da Uniandrade, Curitiba - PR, Brasil.

Autor para correspondência: vinicius.bednarczuk@hotmail.com
\end{abstract}

\section{RESUMO:}

Medicamento é o principal agente causador de intoxicação em seres humanos no Brasil, ocupando o primeiro lugar nas estatísticas do Sistema Nacional de Informações TóxicoFarmacológicas (SINITOX) desde 1994; o que assinala a necessidade de se verificar a ocorrência de intoxicações medicamentosas infantis no estado do Paraná, uma vez que o medicamento é o principal agente causador de intoxicação em seres humanos no Brasil, também se pretende analisar os dados notificados por intoxicação por medicamentos entre crianças, identificar os medicamentos que mais causam intoxicação, identificar os motivos pelos quais ocorreram às intoxicações (negligência dos pais, acidentes), avaliar o número de óbitos infantis, determinar quais as consequências das intoxicações infantis bem como identificar medidas preventivas para intoxicação por medicamentos entre crianças. Tratase de um estudo bibliográfico a partir livros e de artigos obtidos nas bases de dados eletrônicas disponíveis, com foco no descritor nuclear em intoxicação infantil e descritor complementar intoxicação medicamentosa. Os dados foram coletados no Sistema Nacional de Informações Tóxico-Farmacológicas - SINITOX e Sistema de Informação de Agravo de Notificação - SINAN. Verificou-se que o grupo de crianças atingidas de 1 a 4 anos foi a que apresentou maior índice de intoxicação, onde medidas devem ser tomadas, como orientação e armazenamento correto.

Palavras-chave: intoxicação, criança, uso irracional de medicamentos, acidente.

\section{ABSTRACT:}

Drug is the main agent causing intoxication in humans in Brazil, ranking first in statistics of the National System of Toxic-Pharmacological Information (SINITOX) since 1994; which indicates the need to verify the occurrence of children's drug intoxications in the state of Paraná, since the drug is the main agent that causes intoxication in humans in Brazil,.., it is also intended to analyze the data reported for drug intoxication between Identify the causes of poisoning (parental negligence, accidents), assess the number of infant deaths, determine the consequences of child poisoning, and identify preventive measures for drug intoxication between children. It is a bibliographical study from books and articles obtained in the available electronic databases, focusing on the nuclear descriptor in children intoxication and descriptor complementary to drug intoxication. The data will be collected in the National System of Toxic-Pharmacological Information - SINITOX and Information System of Notification Aggravation - SINAN. It was verified that the group of children affected from 1 to 4 years was the one with the highest index of intoxication, where measures should be taken, such as orientation, correct storage.

Keywords: intoxication, child, irrational uso of medicines, accident. 


\section{INTRODUÇÃO}

A intoxicação caracteriza-se como uma manifestação clínica de efeito nocivo produzido em um organismo vivo como resultado da interação de um agente tóxico com esse organismo (CHASIN e PEDROSO, 2004).

Os medicamentos são utilizados como forma de prevenir, curar e diminuir as manifestações clínicas de diversas doenças e são essenciais em função dos benefícios que proporcionam, minimizando o sofrimento, melhorando a qualidade e a expectativa de vida dos indivíduos (NEGREIROS, 2006). Medicamento é o principal agente causador de intoxicação em seres humanos no Brasil, ocupando o primeiro lugar nas estatísticas do Sistema Nacional de Informações Tóxico-Farmacológicas (SINITOX) desde 1994; os benzodiazepínicos, antigripais, antidepressivos, anti-inflamatórios são as classes de medicamentos que mais causam intoxicações em nosso País (44\% foram classificadas como tentativas de suicídio e $40 \%$ como acidentes, sendo que as crianças menores de cinco anos $-33 \%$ e adultos de 20 a 29 anos - 19\% constituíram as faixas etárias mais acometidas pelas intoxicações por medicamentos) (BORTOLETTO e BOCHNER, 1999).

As intoxicações medicamentosas são acidentes comuns na infância. Embalagens e líquidos coloridos, comprimidos com formatos que lembram doces, armazenamento inadequado são alguns dos fatores que levam as crianças a ingerirem medicamentos em grandes doses. Por outro lado, o crescimento de informações via internet, levam alguns pais a medicarem seus filhos de forma irrestrita. O uso desnecessário e incorreto de fármacos e o risco de eventos adversos e intoxicações as quais as crianças estão expostas não refletem um uso racional desses medicamentos (OLIVEIRA e ARNAUTS, 2011).

Os acidentes domésticos entre crianças ocorrem com frequência e contribuem para elevar a mortalidade infantil. Nesse contexto, as intoxicações alcançam uma dimensão preocupante e representam um dos principais tipos de acidente envolvendo crianças. Para Bochner (2005), as intoxicações por medicamentos em crianças são ocasionadas por três fatores: inerentes a própria infância, relacionados à sociedade, e os relacionados ao estado. O primeiro reflete a curiosidade natural das crianças, a falta de noção de perigo e o paladar pouco desenvolvido. O segundo deve-se principalmente a automedicação, ao armazenamento inadequado e a falta de orientação em relação ao uso e riscos oferecidos. Ao fator relacionado ao Estado, pode-se citar o difícil acesso aos centros de saúde e a criação de leis que possibilitem a fiscalização mais rigorosa.

As intoxicações por medicamentos merecem, maior destaque por parte dos 
sistemas de Vigilância em Saúde, de modo a melhor acompanhar o impacto social ocasionado por este evento. O conhecimento do perfil epidemiológico das intoxicações medicamentosas nos municípios e unidades federativas, é importante guia para a gestão de recursos destinados ao planejamento e a implementação de ações que previnam a ocorrência desse agravo (SILVA, 2009). Levando em consideração este contexto, o estudo busca verificar por meio de dados disponibilizados pelos órgãos estaduais e federais a ocorrência de intoxicações medicamentosas infantis no estado do Paraná.

\section{METODOLOGIA}

Esta pesquisa constituir-se-á em um estudo bibliográfico, uma revisão de literatura a partir livros e de artigos obtidos nas bases de dados eletrônicas google acadêmico, Scielo, Pubmed e Science Direct, com foco no descritor nuclear em intoxicação infantil e descritor complementar intoxicação medicamentosa, tendo o público alvo, composto por crianças de 0 a 12 anos. O recorte temporal abrangerá o período de janeiro de 2012 a dezembro de 2015. Também foram utilizados dados do Sistema Nacional de Informações TóxicoFarmacológicas-SINITOX e Sistema de Informação de Agravo de Notificação - SINAN que corresponderão ao ano de 2015. Os dados serão analisados por estatística descritiva, sendo as frequências dispostas em tabelas e gráficos.

\section{REFERENCIAL TEÓRICO}

\subsection{INTOXICAÇÃO}

O SINITOX utiliza a terminologia "intoxicação" para designar episódios com conceituação diversa, porém intoxicação é a manifestação, através de sinais e sintomas, dos efeitos nocivos produzidos em um organismo vivo como resultado da sua interação com substância exógena de potencial tóxico (ZAMBOLIM et al., 2008).

A propriedade intrínseca que o agente tóxico apresenta em causar efeitos nocivos ao organismo denomina-se toxicidade. Esta depende da dose ou concentração, das propriedades físico-químicas da substância, da via de administração, tempo e frequência de exposição e da suscetibilidade do organismo (CHASIN e PEDROSO, 2004).

A intoxicação pode ocorrer, dentre as inúmeras situações, como consequência da exposição aguda ou crônica de substâncias químicas exógenas encontradas no ambiente, 
ar, água, alimentos, plantas, animais, ou isoladas, como pesticidas, medicamentos, produtos de uso industrial e uso domiciliar, entre outros (SCHVARTSMAN e SCHVARTSMAN, 1999).

Efeitos tóxicos locais dependem apenas de ocorrência de contato e tamanho da dose; já os sistêmicos dependem de absorção, distribuição, biotransformação e excreção do agente tóxico (MATOS e NASCIMENTO, 2008).

\subsection{INTOXICAÇÃO POR MEDICAMENTOS}

Os medicamentos possibilitam solucionar vários problemas de saúde, minimizando o sofrimento, melhorando a qualidade e aumentando a expectativa de vida dos indivíduos, mas também tem contribuído para o aumento dos custos em saúde e com o surgimento de agravos à mesma (NEGREIROS, 2006).

O grande número de medicamentos existentes favorece o surgimento de problemas relacionados a estes produtos, como náusea, vômito, irritações de pele, entre outros, representando um risco à saúde pública em países desenvolvidos, assim como nos países em desenvolvimento, como o Brasil (BERTASSO-BORGES et al., 2010).

Segundo Alcântara, Vieira e Albuquerque (2003), a exposição do organismo humano a uma substância química, origina interações que resultam em reações benéficas, adversas ou, quando em doses excessivas, em casos de intoxicações. Portanto, não se deve pensar que os medicamentos para tosse, resfriado e outras patologias frequentes são inofensivos e podem ser tomados sem prescrição e orientação. Muito pelo contrário, esses tratam a patologia, ocasionam efeitos colaterais e, em doses excessivas ou sem a orientação adequada, podem prejudicar o tratamento, induzir a complicações indesejáveis e irreversíveis.

\footnotetext{
"Intoxicação medicamentosa consiste em uma série de sinais e sintomas produzidos, quando um medicamento é ingerido, inalado, injetado ou entra em contato com a pele, olhos ou membranas mucosas em dose(s) acima da(s) terapêutica(s). As intoxicações medicamentosas podem ser classificadas como agudas ou crônicas e cada droga apresenta um quadro de sinais e sintomas peculiares, de acordo com suas características específicas, incluindo a toxicocinética" (MALAMAN et al., 2009).
} 
Na exposição profissional ou acidental, abuso, tentativa de suicídio e homicídio são as circunstâncias que podem levar a um quadro de intoxicação (SILVA, SOUZA e MARQUES, 2011).

Outros fatores que contribuem significativamente para esse quadro são: a frágil regulação da publicidade acerca do medicamento, a facilidade na aquisição de fármacos sob prescrição médica, a deficiência de legislação específica sobre embalagens seguras, iniciativas de desenvolvimento da atenção farmacêutica, e o padrão do consumo de medicamentos pela população, caracterizado pela automedicação, poli farmácia, uso indevido e indiscriminado, principalmente de psicotrópicos e antibióticos (MOTA et al., 2012).

O amplo consumo de medicamentos sem orientação médica tornou-se importante problema de saúde pública, por constituir um dos fatores condicionantes de inibição de condutas preventivas (ALCÂNTARA, VIEIRA e ALBUQUERQUE 2003).

Desta forma, as intoxicações medicamentosas surgem devido a mecanismos complexos, que podem estar relacionados a características do indivíduo, a processos farmacodinâmicos e farmacocinéticos, a propriedades farmacêuticas do produto, a interações medicamentosas ou com outras substâncias e, ainda, ao modo de uso. Assim, o que difere o evento tóxico de uma reação adversa é o fato de naquele, o dano biológico ocorrer, na maioria das vezes, por exposição a doses excessivas (NÓBREGA, COSTA e MARIZ, 2015).

Devido à importância e ao impacto que os medicamentos exercem sobre as pessoas que em quase sua totalidade fazem ou irão fazer uso em algum momento de suas vidas podendo este trazer tanto benefícios como prejuízo à sua saúde é imprescindível colocálos em pauta e discutir a realidade do problema das intoxicações por esses produtos que passam por aspectos legislativos, comerciais e envolve pacientes, propagandistas, profissionais de saúde, formuladores de políticas públicas, indústria e governo sendo este último, através da vigilância sanitária, de importância vital no papel de incitar ações públicas para a melhoria de sua comercialização e utilização e criar e aprovar leis para garantir seu cumprimento (VIEIRA e CAVEIÃO, 2016).

\subsection{GRUPOS DE RISCO}

Vários autores apontam como principais grupos de risco para intoxicações por medicamentos crianças e idosos, por apresentarem alguns fatores específicos, transformando-os em um grupo vulnerável (NEGREIROS, 2006; MASTROIANNI et al., 2011). 
Risco é definido como a probabilidade de ocorrência de uma doença, agravo, óbito ou condição relacionada à saúde, em um grupo ou população, durante um determinado período de tempo. No campo da Toxicologia, é a probabilidade de o indivíduo se intoxicar quando exposto aos diversos agentes químicos que o cercam; e fatores associados ao risco são elementos relacionados ou que tiveram uma associação significativa com a intoxicação (SCHVARTSMAN e SCHVARTSMAN, 1999).

Crianças fazem parte do grupo de maior risco para intoxicações devido a seu comportamento curioso e exploratório: de acordo com a sua fase de desenvolvimento, as crianças levam tudo o que encontram à boca, o que aumenta sua exposição aos agentes que poderão causar inúmeras intoxicações.

Elas são as mais vulneráveis, uma vez que a fase que se encontram denota seu crescimento e desenvolvimento, e assim estão constantemente explorando e investigando tudo que as cerca. Ao explorar o mundo ao seu redor, ocorre aprendizagem. Isso faz com que se exponham a muitos riscos, principalmente a ingestão de produtos potencialmente tóxicos. No decorrer de seu desenvolvimento aumentam a curiosidade, a mobilidade, o que as levam a alcançar medicamentos, produtos domiciliares e outros produtos químicos que estejam armazenados em locais inapropriados (FONTENELE et al., 2015).

Assim como as crianças, os idosos também estão inseridos num grupo de risco que os torna susceptíveis aos efeitos adversos e intoxicações medicamentosas, pois representam um grupo de pessoas que, geralmente, exibem um número maior de doenças, necessitando assim de uma grande quantidade de medicamentos para o tratamento das enfermidades (MASTROIANNI et al., 2011).

\subsection{INTOXICAÇÃO EM CRIANÇAS}

As intoxicações que ocorrem com maior frequência nas crianças são causadas por acidentes com medicamentos, e a maior porcentagem destas, ocorre no sexo masculino, em virtude de que a população apresenta menor precaução com os meninos, deixando-os mais vulneráveis às exposições tóxicas. Com relação às crianças é importante que a família tenha conhecimento que as doses medicamentosas estão relacionadas, em sua ação e toxidade, com o peso da criança e dose administrada (ALCÂNTARA, VIEIRA e ALBUQUERQUE 2003).

Uma das razões para um alto índice de intoxicações entre as crianças se dá nas particularidades do desenvolvimento infantil, na fase oral a criança tem o comportamento 
de levar o que se encontra ao alcance à boca. Nas residências, local de maior ocorrência de intoxicação acidental em crianças, os medicamentos de uso pessoal da família são estocados indevidamente, ficando de fácil acesso a elas (MAIOR e OLIVEIRA, 2012).

Somado a isso se tem a apresentação dos medicamentos, as quais são por diversas vezes coloridas, decoradas e algumas de sabor palatável que atraem a atenção das crianças que podem vir a ingeri-los (BERTASSO-BORGES et al., 2010).

Perante essas circunstâncias, torna-se imprescindível distinguir os fatores que induzem intoxicações em crianças, pois, no decorrer das mudanças fisiológicas que ocorrem, é que se torna indispensável absoluto cuidado pelos pais, visto que é nesse período que despertam as maiores curiosidades pelo desconhecido, e tudo o que enxergam dirigem à boca, e como consequência pode levar a criança até a morte em função da má utilização de qualquer medicamento (ALCÂNTARA, VIEIRA e ALBUQUERQUE 2003).

Os dados de intoxicação infantil dos anos de 2013, 2014 e 2015 podem ser visualizados na figura 1.

\begin{tabular}{|l|r|r|r|r|}
\hline \multicolumn{4}{|c|}{ Dados de Intoxicação infantil por medicamentos no Paraná } \\
\hline & $\mathbf{2 0 1 3}$ & $\mathbf{2 0 1 4}$ & $\mathbf{2 0 1 5}$ & Total \\
\hline $\mathbf{1}$ a 4 anos & 498 & 472 & 281 & 1251 \\
\hline $\mathbf{5}$ a 9 anos & 121 & 122 & 73 & 316 \\
\hline 10 a 14 anos & 262 & 225 & 139 & 626 \\
\hline
\end{tabular}

Fonte: Ministério da Saúde/SVS - Sistema de Informação de Agravos de Notificação - Sinan Net

Figura 1- Dados de Intoxicação infantil por medicamentos no Paraná

Conforme pode ser visualizado na figura 1, observa-se que houve uma diminuição significativa dos casos de intoxicação infantil por medicamentos, na faixa etária de 1-4 anos. Uma das razões para um alto índice de intoxicações entre as crianças se dá nas particularidades do desenvolvimento infantil. Nas residências, os medicamentos de uso pessoal são guardados em lugares inadequados, ficando de fácil acesso a elas (MAIOR e OLIVEIRA, 2012). A apresentação dos medicamentos, por diversas vezes coloridas, atraem a atenção das crianças que podem vir a ingeri-los (BERTASSO-BORGES et al., 2010).

Quando analisamos a idade entre 5 a 9 anos também nota-se relevante diminuição dos casos de intoxicação por medicamentos. Torna-se imprescindível distinguir os fatores 
que induzem intoxicações em crianças, pois, no decorrer das mudanças fisiológicas que ocorrer, é que se torna indispensável o cuidado (ZAMBOLIM et al., 2008).

Conforme pode-se observar no figura 2 a tendência é a diminuição dos dados de intoxicação infantil conforme o avançar da idade, devido a maior compreensão do que é certo ou errado. Observa-se também que os dados de intoxicação no Paraná tem diminuido nos ultimos anos.

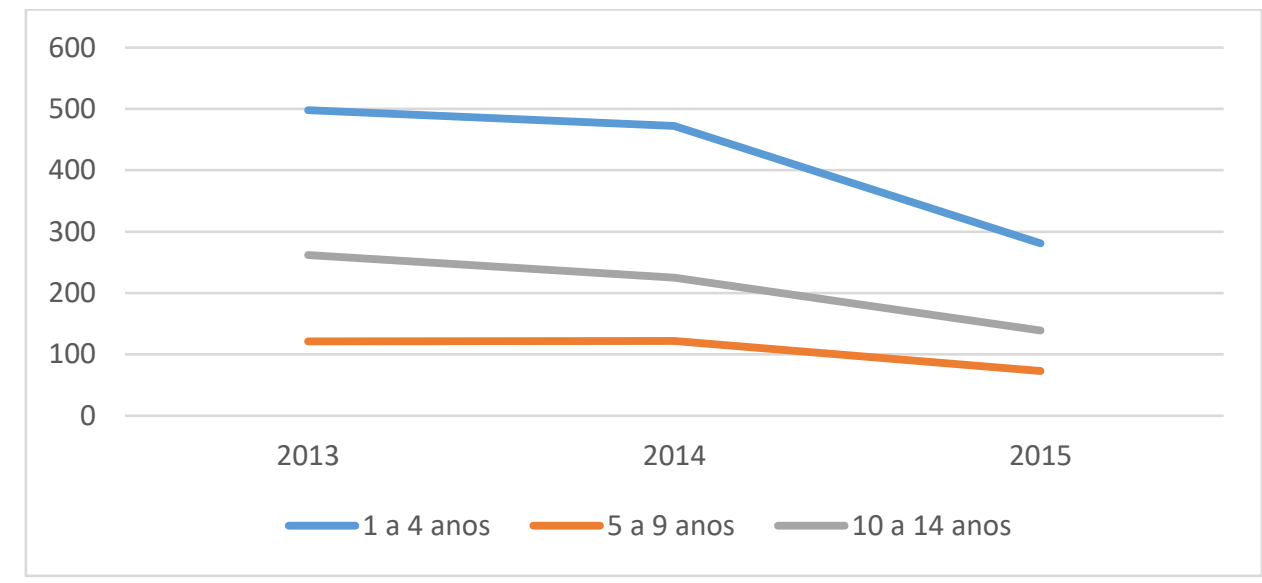

Figura 2 - Intoxicação infantil entre 2013 a 2015.

Entre 10 a 14 anos indica período comprovadamente de risco com relação ao uso de substâncias psicoativas e danos eventualmente associados a este consumo. Por mais que os dados de intoxicação diminuam conforme a idade, o acompanhamento no uso de substâncias com potencial psicoativo deve ser acompanhado.

$\mathrm{Na}$ base SINITOX não há nenhum registro de Casos, Óbitos e Letalidade de Intoxicação Humana por Medicamentos referentes ao estado do Paraná nos anos de 2013, 2014 e 2015.

A inexistência ou o menor número de casos de intoxicações e envenamentos registrados nas estatísticas publicadas pelo SINITOX, nos últimos anos, ocorreu em virtude da diminuição da participação dos Centros de Informação e Assistência Toxicológica (CIATs) nesses levantamentos. Portanto o número de casos de intoxicação e envenenamerntos não vem decrescendo, apenas não estão sendo registrados. 


\section{CONSIDERAÇÕES FINAIS}

A intoxicação por medicamentos é preocupante em qualquer faixa etária, porém as crianças são um grupo de risco devido a curiosidade e a falta de conhecimento, desta forma, é de fundamental importância que o ensino sobre o uso de medicamentos seja realizado desde os primeiros anos de vida, mostrando a importância e o perigo que os medicamentos podem trazer.

Ao rever os dados é possível entender que são necessárias ações de prevenção, uma vez que estas evitariam danos à criança.

O profissional farmacêutico dentro de suas habilitações é um profissional capacitado para prestar assistência, cujo objetivo principal é orientar e conscientizar o paciente que os medicamentos além solucionar vários problemas de saúde, também podem ser prejudiciais a mesma, com a utilização incorreta e irrestrita. Cabe então ao farmacêutico orientar os pais e responsáveis sobre o perigo da exposição acidental das crianças ao medicamento, evitando assim casos de intoxicação que possam acarretar problemas severos ou até mesmo levar a óbito.

\section{REFERÊNCIAS}

ALCÂNTARA, D. A.; VIEIRA, L. J. E. S.; ALBUQUERQUE, V. L. M. Intoxicação medicamentosa em criança. Revista Brasileira em Promoção da Saúde, Fortaleza, v. 16, n. 1-2, p. 10-16, jun. 2003.

BERTASSO-BORGES MS, RIGETTO JG, FURINI AAC, GONÇALVES RR. Eventos toxicológicos relacionados a medicamentos registrados no CEATOX de São José do Rio Preto, no ano de 2008. ArqCiênc Saúde. v. 17, n. 1, p. 35-41, 2010.

BOCHNER, R. Papel da Vigilância Sanitária na prevenção de intoxicações na infância. REVISA, São Paulo, v. 1, n. 1, p. 50-57, jan. 2005.

BORTOLETTO, M. E.; BOCHNER, R. Impacto dos medicamentos nas intoxicações humanas no Brasil. Cadernos de Saúde Pública, Rio de Janeiro, v. 15, n. 4, p. 859-869, 1999.

CHASIN, A. A. M.; PEDROZO, M. F. M. O. Estudo da toxicologia. In: AZEVEDO, F. A.; CHASIN, A. A. M. (Org.). As bases toxicológicas da ecotoxicologia. São Paulo: Rima, 
2004. p. 01-25.

FONTENELE, Gessica Chaves; NOBRE, Micael Pereira; PRADO, Regilane Matos da Silva; SANTOS, Sandna Larissa Freitas dos; TORRES, Karla Bruna.Análise de Intoxicações em Crianças Atendidas no Centro de Assistência Toxicológica do Estado do Ceará. Boletim Informativo Geum, v. 6, n. 1, p. 33-42, 2015

MAIOR, M.C.L.S; OLIVEIRA, N.V.B.V de. Intoxicação medicamentosa infantil: um estudo das causas e ações preventivas possíveis. Rev. Bras. Farm. v. 93, n. 4, p. 422-430, 2012

MALAMAN, K. L.; PARANAÍBA, A. S. C. ; DUARTE, C. M. S.; CARDOSO, R. A. Perfil das Intoxicações Medicamentosas no Brasil. Revista Infarma, v.21, n 7/8, 2009. p. 9-15.

MASTROIANNI, P. C, et. al. Estoque doméstico e uso de medicamentos em uma população cadastrada na estratégia saúde da família no Brasil. Revista Panam Salud Publica. v. 29, n.5, p.358-64. 2011.

MATOS, G.C; NASCIMENTO, A.C. Impacto dos medicamentos como agentes de intoxicações humanas. Revista Racine. v. 106, n. 5, p 59-66, 2008.

MOTA, D. M.; MELO, J.R.R; FREITAS, D.R.C; MACHADO; M. Perfil da mortalidade por intoxicação com medicamentos no Brasil , 1996-2005: retrato de uma década. CienSaude Colet. v.17, n. 1, p. 61-70, 2012.

NÓBREGA HOS, COSTA AMP, MARIZ SR, Fook SML.Intoxicações Por Medicamentos: Uma Revisão Sistemática Com Abordagem Nas Síndromes Tóxicas. REVISTA SAÚDE E CIÊNCIA Online, v. 4, n. 2, p.109-119, 2015.

NEGREIROS, R. L. Agravos provocados por medicamentos em crianças até 12 anos de idade, no Estado do Rio de Janeiro, entre os anos 2000 e 2001. 2006. $61 \mathrm{f}$. Dissertação (Mestrado em Saúde da criança e do Adolescente) - Universidade Federal Fluminense, Niterói, 2006.

OLIVEIRA M.L.F, ARNAUTS I. Intoxicação alcoólica em crianças e adolescentes: dados de um centro de assistência toxicológica. Esc Anna Nery, v. 15, n. 1, p. 83-9, 2011. 
SILVA I. G. SUS como fonte para o estudo de intoxicações causadas por medicamentos no estado do Rio de Janeiro de 1999 a 2007. [Dissertação]. Rio de Janeiro: Programa de Pós Graduação em Saúde Coletiva, Universidade do Estado do Rio de Janeiro, 2009.

SINAN - SISTEMA DE INFORMAÇÃO DE AGRAVOS DE NOTIFICAÇÃO http://portalsinan.saude.gov.br/

SINITOX- SISTEMA NACIONAL DE INFORMAÇÕES TÓXICO-FARMACOLÓGICAS. Disponível em: http://sinitox.icict.fiocruz.br/

SCHVARTSMAN, C.; SCHVARTSMAN, S. Intoxicações exógenas agudas. J. Ped. v. 75, n. 2, p. 244- 50, 1999.

SILVA, C.C.S; SOUZAK.S, MARQUES, M.F.L. Intoxicações Exógenas: Perfil dos casos que necessitaram de assistência intensiva em 2007. Revista BrasCi Saúde. v. 15, n. 1, p. 6568, 2011.

VIEIRA, D. M.; CAVEIÃO, C. Perfil das Intoxicações Medicamentosas no Estado De São Paulo Na Perspectiva da Vigilância Sanitária. Revista Saúde e Desenvolvimento, v. 9, n.5, p. 119-141, 2016.

ZAMBOLIM, C.M; OLIVEIRA, T.P; HOFFMANN, A.N; VILELA, C.E.B; NEVES, D. \& ANJOS, F.R. Perfil das intoxicações exógenas em um hospital universitário. Rev. Med. Minas Gerais. v. 18 , n. 1 , p. $5-10,2008$ 